\title{
System size effects on the mechanical response of cohesive-frictional gran- ular ensembles
}

\author{
Saurabh Singh ${ }^{1, \star}$, Ramesh Kannan Kandasami ${ }^{1, \star \star}$, Rupesh Kumar Mahendran ${ }^{2, \star \star \star}$, and Tejas Murthy ${ }^{1, \star \star \star \star}$ \\ ${ }^{1}$ Indian Institute of Science, Bangalore, India - 560012 \\ ${ }^{2}$ Indian Institute of Technology Madras, Chennai, India - 600036
}

\begin{abstract}
Shear resistance in granular ensembles is a result of interparticle interaction and friction. However, even the presence of small amounts of cohesion between the particles changes the landscape of the mechanical response considerably. Very often such cohesive frictional (c- $\phi$ ) granular ensembles are encountered in nature as well as while handling and storage of granular materials in the pharmaceutical, construction and mining industries. Modeling of these c- $\phi$ materials, especially in engineering applications have relied on the oft-made assumption of a "continua" and have utilized the popular tenets of continuum plasticity theory. We present an experimental investigation on the fundamental mechanics of $c-\phi$ materials specifically; we investigate if there exists a system size effect and any additional length scales beyond the continuum length scale on their mechanical response. For this purpose, we conduct a series of 1-D compression (UC) tests on cylindrical specimens reconstituted in the laboratory with a range of model particle-binder combinations such as sandcement, sand-epoxy, and glass ballotini-epoxy mixtures. Specimens are reconstituted to various diameters ranging from $10 \mathrm{~mm}$ to $150 \mathrm{~mm}$ (with an aspect ratio of 2) to a predefined packing fraction. In addition to the effect of the type of binder (cement, epoxy) and system size, the mean particle size is also varied from 0.5 to 2.5 $\mathrm{mm}$. The peak strength of these materials is significant as it signals the initiation of the cohesive-bond breaking and onset of mobilization of the inter particle frictional resistance. For these model systems, the peak strength is a strong function of the system size of the ensemble as well as the mean particle size. This intriguing observation is counter to the traditional notion of a continuum plastic typical granular ensemble. Microstructure studies in a computed-tomograph have revealed the existence of a web patterned 'entangled-chain' like structure, we argue that this ushers an additional length scale as well as presents a system size effect.
\end{abstract}

\section{Introduction}

In nature, granular materials when stored or under the action of natural forces, develop inter particle cohesion. They develop organic or inorganic bonds between the particles, which changes the mechanical behaviour at the ensemble significantly. These materials acquire their strength from cohesive bonds, in addition to the frictional resistance due to interaction at contacts. For engineering of these materials, i.e. to understand the mechanical behaviour, weak c- $\phi$ granular materials are treated as a "continua" with no consideration of dependency on length scales [1-4]. In case of brittle and quasi-brittle materials such as glass, rock, concrete etc. scaling is well understood and is considered in any numerical modeling which is inspired from Weibull's statistical model [5]. It is well known that the fabric or structure of c- $\phi$ granular materials change with changes in amount of cohesion/binder present [10]. This proportion of binder and the arrangement of particles due to the presence of binders, brings

\footnotetext{
^e-mail: saurabh@civil.iisc.ernet.in

$\star \star$ e-mail: ramesh.k.kannan@gmail.com

$\star \star \star$ e-mail: dmrupeshkumar@gmail.com

$\star \star \star \star$ e-mail: tejas@ civil.iisc.ernet.in
}

forth fabrics in c- $\phi$ materials such as a matrix bound structure (asphalt - wherein individual particles are suspended in a matrix of the binder), void bound structure (concrete, mortar, rocks - wherein the voids in-between particles are filled with the binder) and contact bound structure(weak c- $\phi$ materials, geological materials such as sandstones). It has been widely accepted that materials with void bound structure follow Weibull's statistical scaling model, in that with increase in the size of the system (or specimen) the strength reduces and has been well adapted into the mechanics and design of such systems. In this study we seek to understand if scaling other than the continuum scaling exists in weak c- $\phi$ materials (i.e. materials with a contact bound structure) with varying system size and particle size.

\section{Background}

In order to model weakly cohered frictional particulate ensembles (in short c- $\phi$ materials), two broad approaches have been practiced. In the first approach, models for frictional granular materials were used and modified to incorporate additional features exhibited due to the presence of 
a weak cohesion between the particles. In order to accomplish this, utilising the framework of the mathematical theory of continuum plasticity, the features of a plastic model are modified, i.e. the plastic potential function, yield function are modified to take care of cohesion by considering this cohesion as an additional confinement on the ensemble. In other words, the yield surface and plastic potential surfaces were translated along hydrostatic axis to include this cohesion or cementation in the material [3,4]. While another approach has been less phenomenological, by observing the governing mechanism and the evolving mechanism in the inter particulate cohesion to reach failure [1,2]. In a typical study of this kind, [1,2] assumed that net load taken by the ensemble can be thought as a combination of resistance of particles at the contact and bonds which is true at both micro (inter particle) and macro (ensemble or continuum) levels. Separate elastic-plastic evolution laws were considered for two phases and then combined using the micro-mechanical equilibrium. The model parameters for such constitutive models were obtained by performing "continuum" elemental tests on cylindrical specimens of standard dimension $(38 \times 76 \mathrm{~mm})$. These elemental tests can be relied upon to provide an appropriate response of the material only if the response is that of a true or a typical "continuum material". In that, the overall mechanical response along with failure strength is not affected by changing the size of the system under consideration. This has been clearly documented through simulations and experiments for the case of a purely frictional granular material [6]. However, thus far, no study has thrown light if such continuum assumptions and scale independence is valid for these weak $c-\phi$ materials (or materials with a contact bound structure). As stated earlier, in case of brittle and quasi brittle materials such as (c- $\phi$ material with void bound structure) concrete, rock show significant scaling in the strength of the material. With increase in specimen size, peak strength (failure strength) decreases. This appears akin to weibull's distribution which was initially coined for failure of a chain like structure with links. Where failure of a link is considered as failure of the entire chain. With increasing number of links probability of failure increases which resembles the behaviour of these quasi-brittle materials. That is, with increase in size of a specimen, the structural strength decreases (or the probability of failure increases). Bazant et al.[7] has provided an extension of this in a framework of fracture mechanics.

In recent times, discrete element simulations for weak c- $\phi$ materials have been carried out [8]. The results show a systematic transition from elastic behaviour followed by bond breakage which in turn leads to dilation, as a result of the frictional rearrangement of the particulates. A change in the stress distribution from web patterned force chains to columnar structure of force chains was also shown from these simulations. A computed tomographic scan image of an initial contact bound structure is shown in the figure 1 .

\section{Experimental}

In these experiments, we used two model granular materials - sand and glass ballotini and two materials for im-

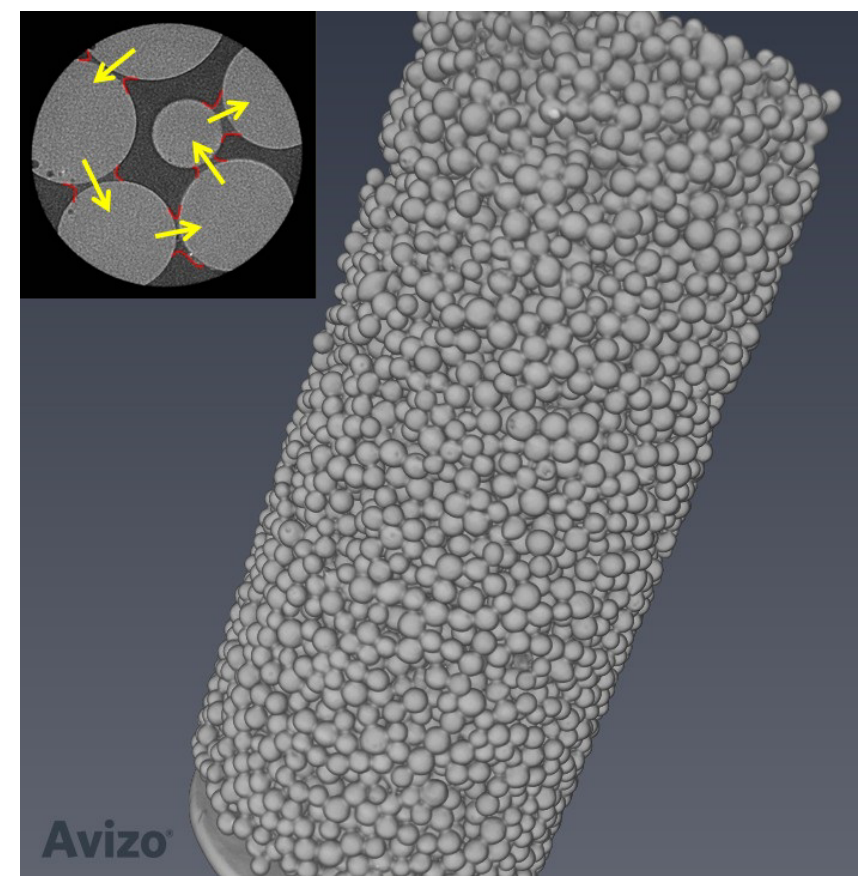

Figure 1. Computed tomography image of a weakly cemented specimen (glass ballotini $+1 \%$ epoxy) showing a contact bound structure. The inset shows the chain like cemented structure

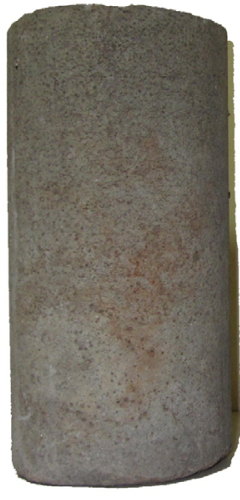

$150 \mathrm{~mm} \phi$

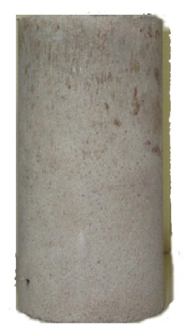

$100 \mathrm{~mm} \phi$

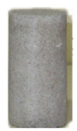

$38 \mathrm{~mm} \phi \quad 20 \mathrm{~mm} \phi \quad 10 \mathrm{~mm} \phi$
Figure 2. Artificially reconstituted weakly cemented (sand $+4 \%$ cement) with specimen/ system size varying from 150 to $10 \mathrm{~mm}$ dia keeping the particle size, density and aspect ratio constant

parting inter grain cohesion - cement and epoxy, in effect, we used three c- $\phi$ systems sand+cement (cemented sand), sand+epoxy (SE) and glass beads+epoxy (GBE).

For the case of cemented sands, specimens were reconstituted by mixing quartzitic sand (specific gravity 2.65 and mean grain size $0.45 \mathrm{~mm}$ ) with ordinary Portland cement (OPC) at a density of $1.4 \mathrm{~g} / \mathrm{cc}$. Three types of cemented sand specimens were prepared using $2 \%, 4 \%$, and $8 \%$ OPC by weight of sand. For each specimen the ratio of height to diameter (aspect ratio) was kept constant at two. To understand the system size effect specimens with diameter of $10 \mathrm{~mm}, 20 \mathrm{~mm}, 38 \mathrm{~mm}, 100 \mathrm{~mm}, 150 \mathrm{~mm}$ were prepared. All the specimens were prepared to a desired 


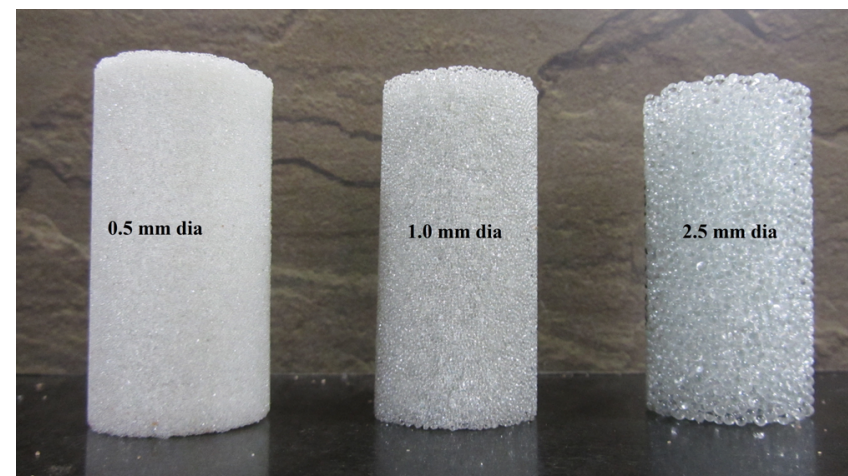

Figure 3. Artificially reconstituted weakly cemented (glass ballotini $+1 \%$ epoxy) specimens with particle size varying from 0.5 to $2.5 \mathrm{~mm}$ dia while keeping the system size and density constant

packing fraction and were cured under moist conditions to achieve a contact bound structure and a characteristic compressive strength. Figure 2 shows the prepared specimen at different diameters.

For GBE, specimens were prepared by thoroughly mixing glass beads (specific gravity 2.5 ) with $1 \%$ epoxy uniformly to create ensembles of density of $1.5 \mathrm{~g} / \mathrm{cc}$. Three types of specimens with particle diameter $0.5 \mathrm{~mm}$, $1.0 \mathrm{~mm}, 2.5 \mathrm{~mm}$ were chosen to understand the particle size effect on the failure strength of weak c- $\phi$ granular materials. Specimens were cured by heating in oven at $50^{\circ} \mathrm{C}$ for 48 hours. Figure 3 shows the specimens prepared to study particle size effect. Similar procedure was followed to prepare SE specimen.

All the uniaxial compression tests were performed at a constant strain rate of $0.5 \%$ per min.

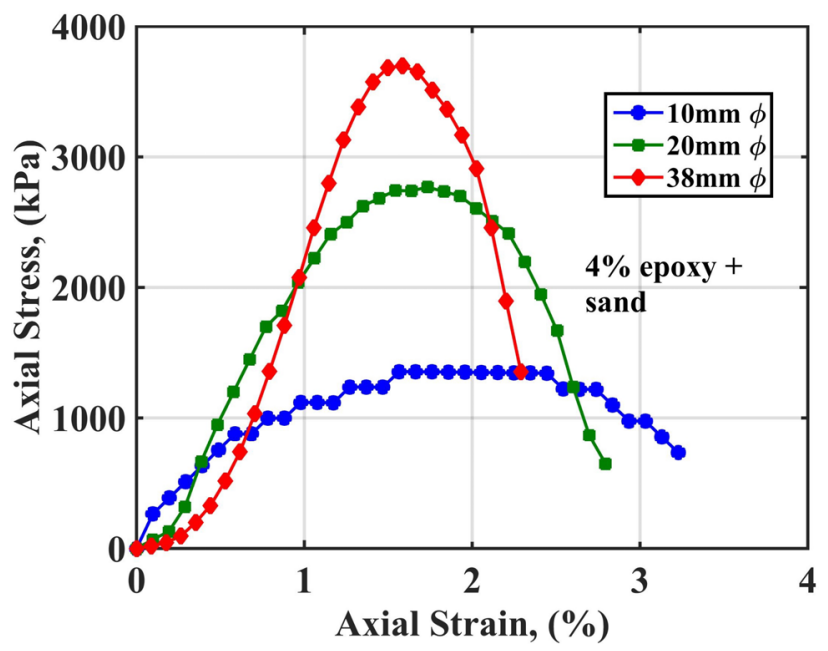

Figure 4. Stress strain response for cemented sand sample with specimen diameters of $10 \mathrm{~mm}, 20 \mathrm{~mm}, 38 \mathrm{~mm}$

\section{Results}

A typical stress-strain plot is shown in figure 4 with varying specimen dimension (or with increasing number of

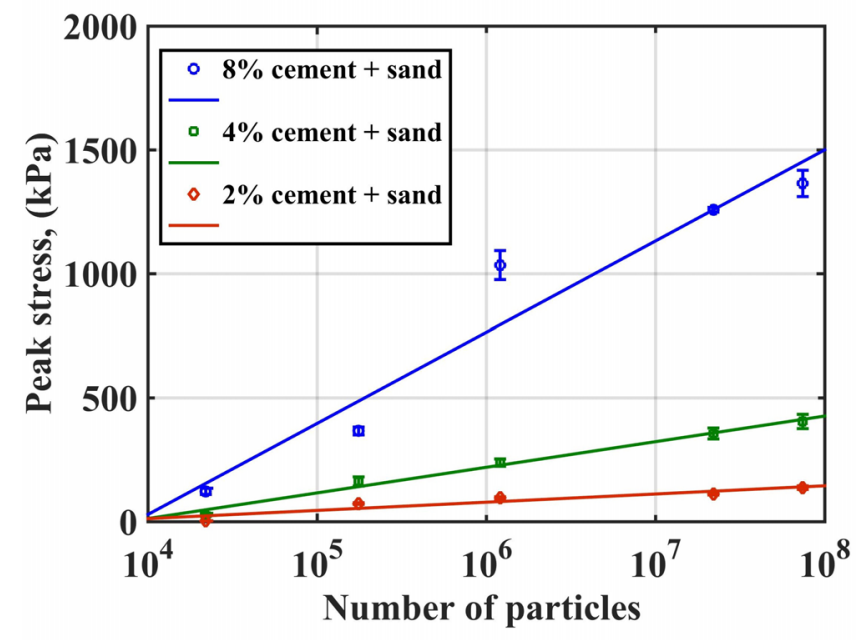

Figure 5. The peak strength increases with increase in the percentage of cementation as well as increase in the number of particles across the cross section or as the system size increases

particles) for SE specimen. The increase in specimen dimension not only reflects in the change of peak strength but also other features of stress behaviour such as elastic stiffness, failure stress, hardening, and softening are significantly affected. Other parameters such as mean particle size $(0.45 \mathrm{~mm})$, density $(1.4 \mathrm{~g} / \mathrm{cc})$, binder content $(4 \%)$ were kept constant for studying system size effect.

This effect of system size on cemented sand specimens was studied for various amounts of cohesion of $2 \%, 4 \%$, $8 \%$, it should be noted that even with increase in the binder content, a contact bound structure was ensured. A plot of peak strength with number of particles (or specimen dimension) is presented in figure 5 along with a linear fit for each binder content. With increase in binder content normalized peak strength with number of particles (slope of fit lines) increases. This linear fit can be interpreted as the number of particles directly controlling the failure strength.

Figure 6 shows a typical stress-strain curve for GBE with $2 \%$ binder content. For understanding the effect of particle size; the density of the ensemble, specimen dimension and binder content were kept constant. With increasing particle diameter the failure strength correspondingly decreases. We refer to this as the particle size effect. We further studied this by varying binder content, however, ensuring a contact based structure. A plot of peak strength is presented for $1 \%$ and $2 \%$ epoxy in figure 7 . The peak strength is shown to increase with increasing number of particles and binder content. The normalized peak strength with number of particles appears to be constant. Similar results were obtained for sand+epoxy specimen with changing mean grain size.

\section{Discussion}

Brown et. al [9] present a set of experiments on granular chains, especially highlighting the shear stiffening of 


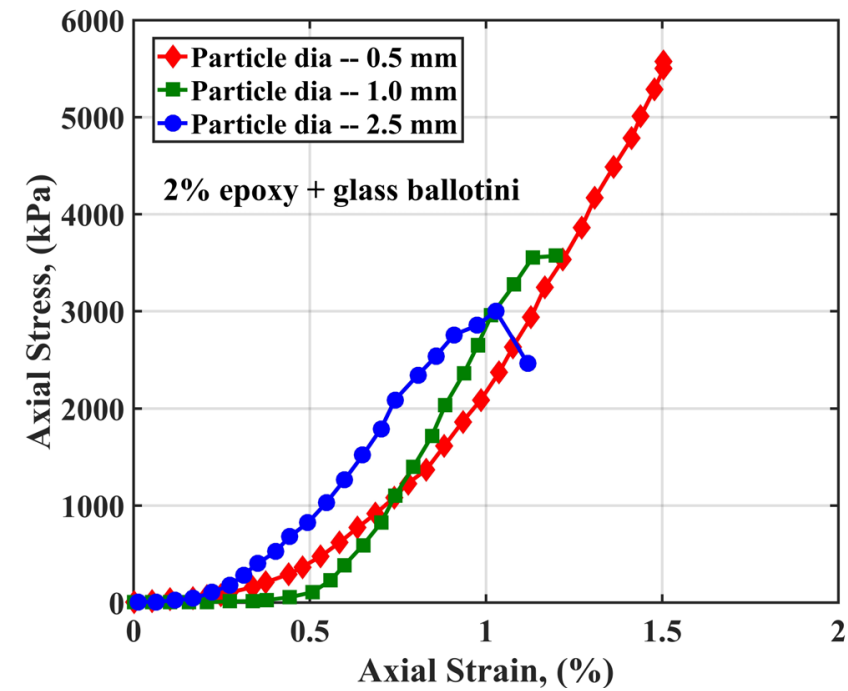

Figure 6. Stress strain response for GBE with changing particle dia for specimen diameter of $38 \mathrm{~mm}$

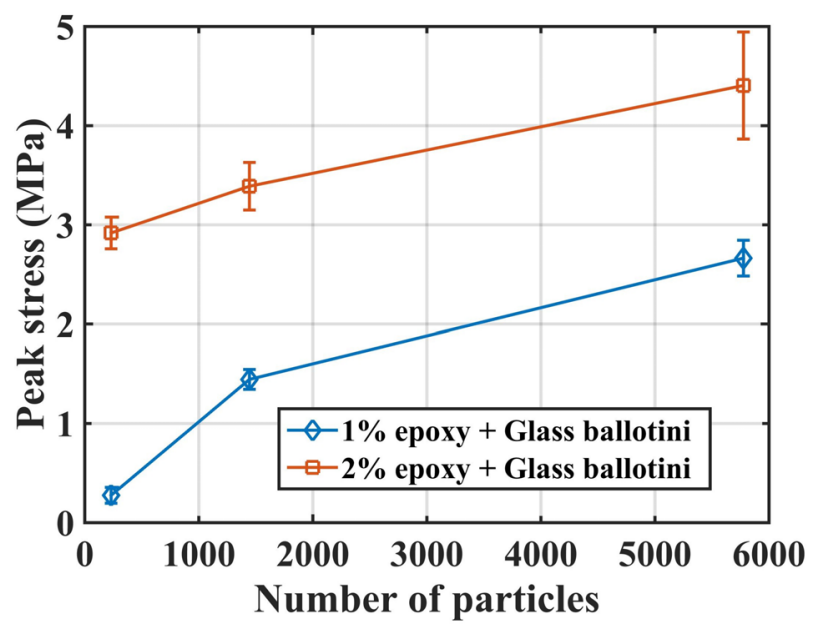

Figure 7. The peak strength increases with increase in the number of particles across the cross section or as the particle size decreases

random packed granular chains. In order to explain the interesting phenomenology of their experiments, the authors suggest that the increase in the length of a granular chain contributes to increased entanglement, in turn contributing to higher shear stiffening (or hardening). A similar increase in failure strength with specimen size (or number of particles) is observed in our experiments. We conjecture that the structure of weak c- $\phi$ materials can be construed as an ensemble comprising of multiple granular chains. Given that the structure of the ensemble is complex, we further suggest that this c- $\phi$ ensemble can be modelled as a series of knotted (entangled) granular chains. Brown et al. [9] further defined a minimum loop circumference (mlc) which was a function of particle diameter. With increase in the particle diameter the minimum loop circumference decreased for a given chain length whereas this minimum loop diameter also increases as the particle size decreased. The entanglement, or the minimum loop circumference governed the overall mechanical behaviour of the ensemble. If the chain length was smaller than mlc then material behaviour resembled a typical granular ensemble. If chain length was sufficiently larger than the mlc then significant strain stiffening was observed within a test along with an increase in peak strength as the chain length increases.

For weak c- $\phi$ materials considered in our experiments, system size or the particle size both affect the length of a "hypothetical granular chain". As the system size increases, we further conjecture that the lengths of these granular chain structures also increases, in other words, the possibility of obtaining more entangled structure increases. Similarly with increase in particle size although the chain length would remain the same, however, the equivalent mlc decreases which further causes reduction in entanglement measure.

Figure 5 and figure 7 represent the system size and particle size effect, respectively. The results are plotted between the peak strength and number of particles to support our argument of minimum loop circumference.

We present exploratory set of experimental results, and the first evidence of the existence of a second length scale beyond the traditional continuum length scale in weakly cohered-granular ensembles. Ongoing work is implementing these systems through extensive tomography for understanding the microstructure, and the manifestation of this microstructure at the ensemble level.

\section{References}

[1] Abdulla, Ali A and Kiousis, Panos D, International Journal for Numerical and Analytical Methods in Geomechanics 21, 549-568 (1997)

[2] Vatsala, A and Nova, R and Murthy, BR Srinivasa, Journal of Geotechnical and Geoenvironmental Engineering 127, 679-687 (2001)

[3] Gao, Zhiwei and Zhao, Jidong, Computers and Geotechnics 41, 57-69 (2012)

[4] Lade, Poul V and Kim, Moon K, International Journal of Solids and Structures 32, 1963-1978 (1995)

[5] Weibull, Waloddi, Journal of applied mechanic 103, 293-297 (1951)

[6] Jefferies, M.G., Been, K. and Hachey, J.E., Canadian Geotechnical Conference 43, (1990)

[7] Bazant, Zdenek P and Xi, Yunping and Reid, Stuart G, Journal of engineering Mechanics 117, 2609-2622 (1991)

[8] Wang, YH and Leung, SC, Canadian Geotechnical Journal 45, 29-44 (2008)

[9] Brown, Eric and Nasto, Alice and Athanassiadis, Athanasios G and Jaeger, Heinrich M, Physical review letters 108, (2012)

[10] Sowers, GB and Sowers, GF, Introductory soil mechanics and foundations (The Macmillan Co., New York, 1961) 26-27 